\title{
Conjunctival biopsy in adult form galactosialidosis
}

\author{
Tomoaki Usui, Shoichi Sawaguchi, Haruki Abe, Kazuo Iwata, Kiyomitsu Oyanagi
}

\begin{abstract}
Conjunctival biopsy was performed in two siblings with adult-form galactosialidosis. Electron microscopically, several types of intracytoplasmic inclusion were observed in the fibroblasts in conjunctival stroma, lymphatic capillary endothelial cells, Schwann cells, and epithelial cells. Membrane-bound vesicles with fibrillogranular content were frequently observed, and occasional lamellar structures were noted in these inclusions. Dense granular inclusions and oil droplets were also seen. Dense granular inclusions have not been reported in this disease previously.
\end{abstract}

(Brf Ophthalmol 1993; 77: 165-167)

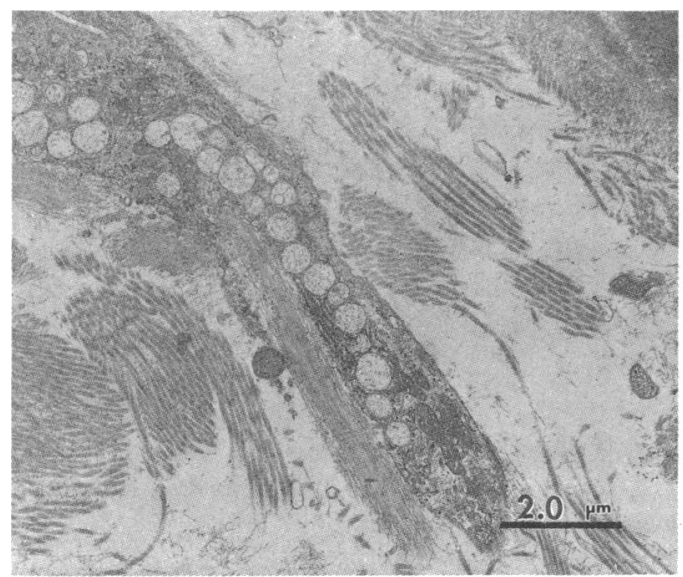

Figure 1 The fibroblast in conjunctival stroma (case 1). Abundant vesicles with some fibrillogranular content were noted.

Department of Ophthalmology, Niigata University School of Medicine, Niigata, Japan T Usui

S Sawaguchi

H Abe

K Iwata

The Center for Materials of Brain Diseases, Brain Research Institute, Niigata University, Niigata Japan K Oyanagi

Correspondence to: Tomoaki Usui, MD, Department of

Ophthalmology, Niigata University School of Medicine, 1 Asahimachi Niigata 951, Japan.

Accepted for publication 10 December 1992

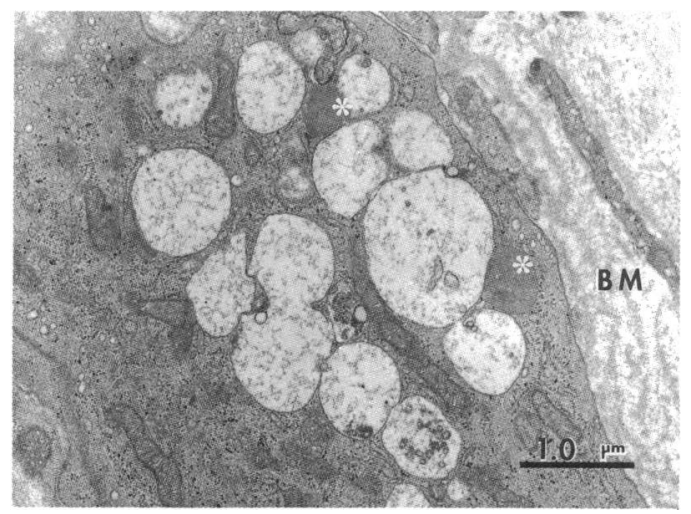

Figure 2 Schwann cell (case 1). Plenty of membrane-bound vesicles were observed. Dense granular materials were also seen in some vesicles (white asterisks). $B M=$ basement membrane.
Galactosialidosis is a lysozomal storage disease characterised by decreased $\beta$-galactosidase and sialidase activity. Clinical features include myoclonus, cerebellar ataxia, epilepsy, mental retardation, angiokeratoma, gargoyle-like facial features, skeletal dysplasia, and visual disturbance. ${ }^{1-3}$ The cause of this disease is thought to be a defect of a 'protective' protein which is necessary for activating both $\beta$-galactosidase and sialidase. ${ }^{47}$ This disease is classified into three types according to age at onset: (1) the early infantile form, (2) the late infantile form, and (3) the juvenile/adult form. ${ }^{8}$ Several reports on conjunctiva in this disease have described variable findings which showed cytoplasmic vacuoles in the fibroblasts, epithelial cells, endothelial and goblet cells, ${ }^{9-13}$ and lamellar structures in the Schwann cells, ${ }^{12}{ }^{13}$ but no other abnormalities. ${ }^{14}$ Agreement on these conjunctival findings has not been reached because of the rarity of this disease. We studied the conjunctiva of two siblings with adult-form galactosialidosis and found a new inclusion body in the conjunctival cells.

\section{Patients and methods}

The patients were a 41-year-old man and a 46year-old woman. Their detailed clinical ophthalmic findings have been described in a previous report (cases 1 and 2). ${ }^{15}$ The onset of disease was when they were teenagers. Their conjunctivas showed mild venous congestion, microaneurysms, and venous dilatation, although these changes might be seen in aging, arteriosclelosis, and vascular hypertension.

Informed consent was obtained from these patients. Biopsies were performed by applying topical oxybuprocaine hydrochloride drops. A piece of bulbar conjunctiva (approximately $1 \times 1 \mathrm{~mm}$ ) was elevated with forceps and excised. Antibiotic ophthalmic ointment was instilled, and the patients were allowed to leave the clinic. No complication or morbidity occurred.

The specimens were fixed in $3 \%$ glutaraldehyde and $1 \%$ paraformaldehyde in a $0.1 \mathrm{~mol} / \mathrm{l}$ phosphate buffer (pH 7.3), post-fixed in $1 \%$ osmium tetroxide, dehydrated through a graded ethanol series, and embedded in epoxy resin (Epon 812). Sections of $1 \mu \mathrm{m}$ thickness were stained with toluidine blue. Ultrathin sections stained with uranyl acetate-lead citrate were examined under the electron microscope (H-7000, Hitachi Instrument Service Co Ltd, Tokyo, Japan). 


\section{Results}

The fibroblasts in conjunctival stroma were filled with abundant vesicles having a fibrillogranular content (Fig 1). Schwann cells also had many membrane-bound vesicles (Fig 2). Dense granular materials were also seen in some vesicles. The conjunctival epithelium had many large membrane-bound vesicles with abundant fibrillogranular content but little lamellar structure (Fig 3). Some fibrillogranular content, dense granular inclusions, and vesicles were seen in the cytoplasm of the conjunctival fibroblast (Fig 4). The stromal fibroblasts appeared filled with vesicles (Fig 5). A few vesicles were filled with dense granular inclusions having lamellar structures. Fibrillogranular structures containing a small number of oil droplets were occasionally seen. Laminated lamellar structures combined with fibrillogranular structures were also seen in the fibroblasts (Fig 6). The lymphatic capillary endothelial cells contained few membrane-bound vesicles. Membrane-bound vesicles in endothelial cells appeared less predominant compared with those seen in the fibroblasts and conjunctival epithelia. Similar findings occurred in both patients.

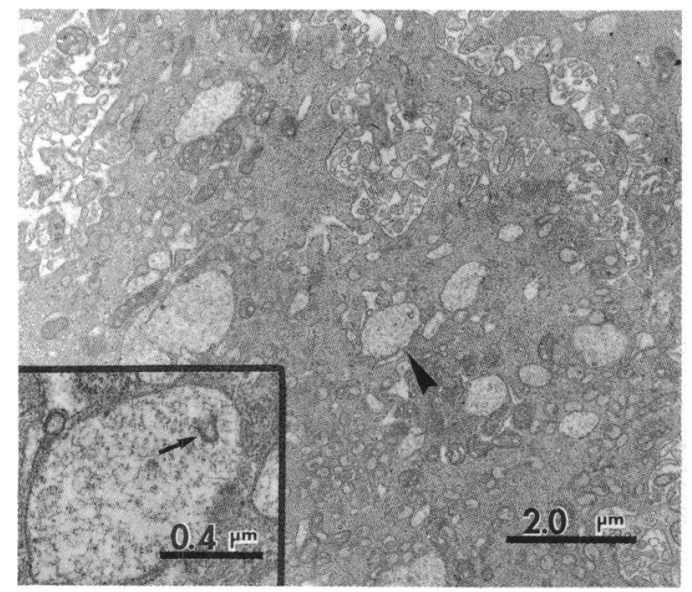

Figure 3 The conjunctival epithelium (case 2). Many large membrane-bound vesicles with an abundant fibrillogranular content were evident. The portion marked by an arrowhead is magnified and shown in the inset. Note the lamellar structure (arrow).

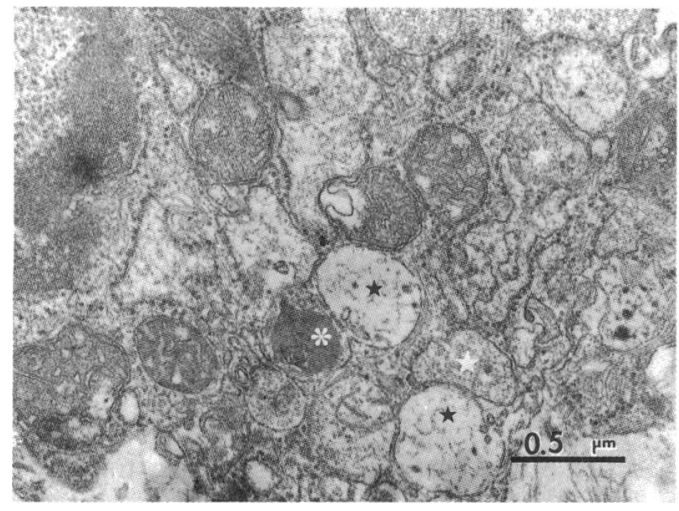

Figure 4 The conjunctival fibroblast (case 2). With fibrillogranular content (black stars), dense granular inclusions (white asterisk), and vesicles (white stars) co-existing in the cytoplasm.

\section{Discussion}

There have been a few reports of conjunctival findings of patients with confirmed deficiencies of both neuraminidase (sialidase) and $\beta$ galactosidase activities. ${ }^{9-13}$ Patients with the adult form have cytoplasmic vacuoles in the fibroblasts, epithelial cells, endothelial and goblet cells, ${ }^{9-11}$ and lamellar structures in the Schwann cells. ${ }^{12}{ }^{13}$ In a case of the late infantile form, Goldberg et $a l^{12}$ reported that no abnormalities in the conjunctival cells were found although a conjunctival biopsy specimen from a subject who was deficient in $\beta$-galactosidase had values $55 \%$ of those of controls.

Our results showed several types of inclusions, including membrane-bound vesicles having a fibrillogranular content, dense granular inclusions, lamellar structure, and oil droplets. Membrane-bound vesicles were frequently observed, however, the other forms were rare. Lamellar structures were occasionally seen within the membrane-bound vesicles. Intracytoplasmic inclusions in the conjunctiva were quite different from those observed in the retina, which had an almost concentric or wavy form, with parallel lamellar inclusions and membrane-

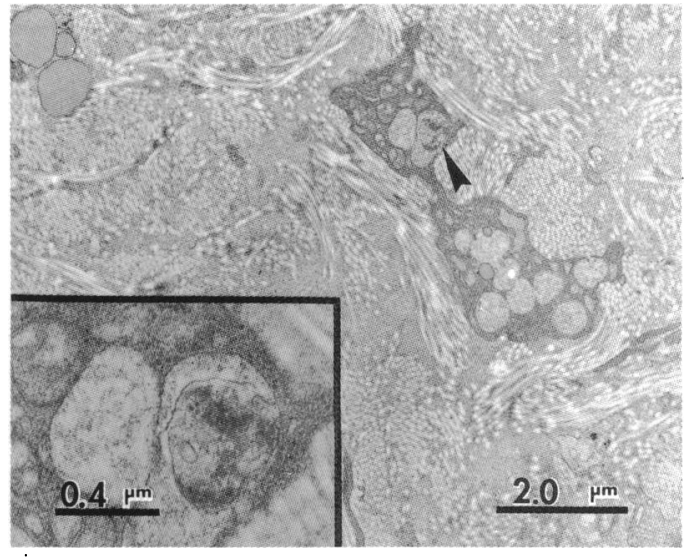

Figure 5 The stromal fibroblast (case 2). A few vesicles were filled with dense granular inclusions having lamellar structures. Fibrillogranular structures with a small number of oil droplets were occasionally seen. Higher magnification of section indicated by arrowhead is given in the inset.

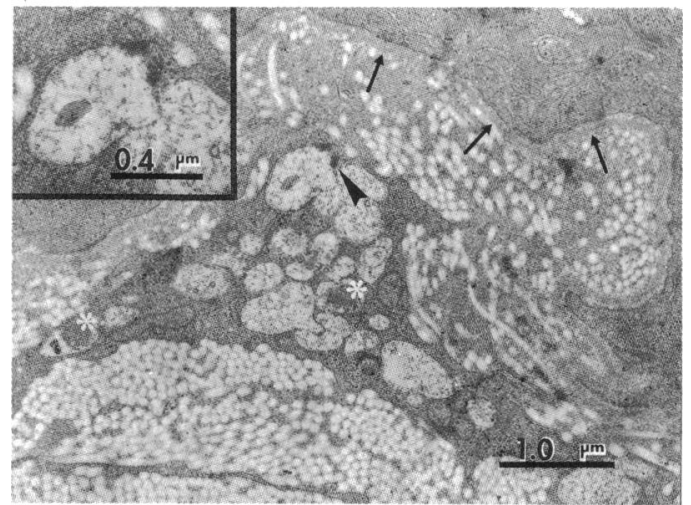

Figure 6 The stromal fibroblast (case 2). The laminated lamellar structure was evident (arrowhead). Asterisks indicate the vesicles with dense granular content. Arrows indicate the basement membrane of the basal epithelium of the conjunctiva. Higher magnification of section indicated by arrowhead is shown in inset. 
bound, partially crossing, and parallel lamellar inclusions. ${ }^{16}$ The membrane-bound vacuole with fibrillogranular content observed in our cases resembled the intraneuronal inclusion seen in the central nervous system (cerebral cortex, brain stem, basal nucleus of Meynert, etc). ${ }^{17}$ The cytoplasmic vacuole and lamellar inclusion seemed to correspond to mucopolysaccharides and lipids, respectively. ${ }^{12}$

Dense granular inclusions have not been reported in this disease before. These storage materials have not been precisely determined in conjunctiva. Further investigation is needed to determine the metabolic mechanisms in the conjunctiva.

1 Andria G, Strisciuglio P, Pontarelli G, Sly WS, Dodson WE Infantile neuraminidase and $\beta$-galactosidase deficiencies (galactosialidosis) with mild clinical courses. In: Tettamanti G, Durand $\mathrm{P}, \mathrm{Di}$ Donato $\mathrm{S}$, eds. Perspectives in inherited metabolic diseases. Vol 4. Sialidases and sialidoses. Milan: Ermes, 1981: 379-95.

2 Lowden JA, O'Brien JS. Sialidosis: a review of human neuraminidase deficiency. Am $\mathcal{F}$ Hum Genet 1979; 31: 1-18. Miyatake T, Atsumi T, Obayashi T, Mizuno Y, Ando S, Ariga $T$, et al. Adult-type neuronal storage disease with neuraT, et al. Adult-type neuronal storage disease with

4 d'Azzo A, Hoogeveen A, Reuser AJJ, Robinson D, Galjaard H. Molecular defect in combined $\beta$-galactosidase and neuraH. Molecular defect in combined $\beta$-galactosidase and neura5 Verheijen FW, Palmeri S, Hoogeveen AT, Galjaard H. Human placental neuraminidase: activation, stabilization and association with $\beta$-galactosidase and its 'protective' protein. Eur $\mathcal{F}$ Biochem 1985; 149: 315-21.

6 Palmeri S, Hoogeveen AT, Verheijen FW, Galjaard H. Galactosialidosis: molecular heterogeneity among distinct clinical phenotypes. Am f Hum Genet 1986; 38: 137-48.

7 Strisciuglio P, Parenti G, Giudice C, Lijoi S, Hoogeveen AT, d'Azzo A. The presence of a reduced amount of $32-\mathrm{kDa}$ 'protective' protein is a distinct biochemical finding in late infantile galactosialidosis. Hum Genet 1988; 80: 304-6.

8 Miyashita K, Miyatani N, Yoshino H, Tsuji S, Miyatake T. Late-infantile form galactosialidosis with psychomotor retardation and spastic paraparesis. Clin Neurol (Tokyo) 1989; 29: 1244-50

9 Tanabe S, Tabuchi Y, Hirano J, Murakami M. A case of galactosialidosis. F pn f Clin Ophthalmol 1987; 41: 824-5.

10 Harada T, Hisada H, Tanabe S, Hoshino T. Lectin histochemistry of conjunctival specimens in some glycolipid storage diseases. Folia Ophthalmol fpn 1988; 39: 1673-9.

11 Ishida M, Hayakawa M, Kanai A, Nakajima A, Mori H. A case of sialidosis type II. F pn f Clin Ophthalmol 1987; 41: 63-7.

12 Mori H, Hayashi A, Sato T. Skin and conjunctival biopsies in adult type $\beta$-galactosidase - neuraminidase deficiency. Clin Neurol (Tokyo) 1985; 25: 776-83

13 Martin JJ, Libert J, Ceuterick C. Conjunctival and skin biopsies in sialidosis. In: Tettamanti G, Durand P, Di Donato S, eds. Perspectives in inherited metabolic diseases. Vol 4. Sialidases and sialidoses. Milan: Ermes, 1981: 341-64.

14 Goldberg MF, Cotlier E, Fichenscher LG, Kenyon K, Enat R, Borowsky SA. Macular cherry-red spot, corneal clouding, and B-galactosidase deficiency. Arch Intern Med 1971; 128: 387-98.

15 Usui T, Takagi $M$, Abe $H$, Iwata $K$, Tsuji S, Miyatake T. Adult-form galactosialidosis: Ocular findings in three cases. Ophthalmologica 1991; 203: 176-9.

16 Usui T, Sawaguchi S, Abe H, Iwata K, Oyanagi K. Lateinfantile type galactosialidosis: histopathology of retina and infantile type galactosialidosis: histopathology of

17 Oyanagi K, Ohama E, Miyashita K, Yoshino H, Miyatake T Yamazaki $\mathrm{M}$, et al. Galactosialiodosis: neuropathological findings in a case of the late-infantile type. Acta Neuropathol 1991; 82: 331-9. 